\title{
DR-19. HYDROTHERMAL SYNTHESIS OF ZnO NANOPOWDER AND ITS PHOTOCATALYTIC PERFORMANCE UNDER UV AND VISIBLE LIGHT IRRADIATION
}

\author{
$\underline{\text { G. Thirumala Rao }}^{1}$, R. V. S. S. N. Ravikumar' ${ }^{2}$, N. Bakthavatchala Reddy ${ }^{3}$, G. V. Zyryanov, \\ ${ }^{1}$ Physics Division, Department of Basic Sciences \& Humanities, GMR Institute of Technology, \\ Rajam, Andhra Pradesh, 532127, India \\ ${ }^{2}$ Department of Physics, Acharya Nagarjuna University, Nagarjuna Nagar, \\ Andhra Pradesh, 522510, India \\ ${ }^{3}$ Ural Federal University of the first President of Russia B. N. Yeltsin, \\ Mira St., 19, Yekaterinburg, 620002, Russia \\ ${ }^{4}$ I. Ya. Postovsky Institute of Organic Synthesis UB RAS, \\ S. Kovalevskoy/Akademicheskaya St., 20/22, Yekaterinburg, 620990, Russia \\ E-mail: thirumalaphy@gmail.com
}

Nanostructured semiconducting materials open up new opportunities in the field of photocatalysis and have an enormous impact on the energy and environmental sectors. Several nanostructured metal oxides including $\mathrm{TiO}_{2}, \mathrm{ZnO}, \mathrm{MoO}_{3}$ and binary metal oxides have been studied for photocatalytic applications. Among them, $\mathrm{ZnO}$ has been extensively investigated for energy conversion devices due to its high carrier mobility, ease of synthesis, and nontoxicity. However, several unfavorable drawbacks, such as a wide bandgap of 3,37 eV and fast internal recombination of charge carriers, lead to low quantum yields and poor solar conversion efficiency. In this regard, it is highly desirable to improve the photocatalytic efficiency of $\mathrm{ZnO}$ under visible light irradiation.

Herein, we reported the hydrothermal synthesis of $\mathrm{ZnO}$ nanopowder for enhanced visible light photocatalytic performance. The prepared nanopowder was characterized by various experimental techniques like Powder XRD, SEM with EDS, TEM, Optical absorption, PL, FT-IR and photocatalytic activity studies. XRD pattern confirmed that the synthesized nanopowder exhibited hexagonal wurtzite structure with the average crystallite size of $16 \mathrm{~nm}$. SEM and TEM micrographs reveal the spherical like structures with little agglomerates. EDS pattern confirmed the purity of the sample by exhibiting only the target elements, Zn and O. Optical absorption spectrum exhibited the characteristic band at $363 \mathrm{~nm}$ due to the excitonic absorption of $\mathrm{ZnO}$. Photoluminescence spectrum exhibits less intense near band edge emission at $381 \mathrm{~nm}$. FT-IR spectrum exhibited symmetric stretching vibrational mode centered at $482 \mathrm{~cm}^{-1}$. The photocatalytic activity of the prepared $\mathrm{ZnO}$ nanopowder has been examined for methylene blue dye under UV and visible light irradiation. 